Research

\title{
Phellodendron and Citrus extracts benefit joint health in osteoarthritis patients: a pilot, double-blind, placebo-controlled study
}

\author{
Julius Oben ${ }^{1}$, Ebangha Enonchong ${ }^{1}$, Shil Kothari ${ }^{2}$, Walter Chambliss ${ }^{3}$, \\ Robert Garrison ${ }^{4}$ and Deanne Dolnick*4
}

Address: ${ }^{1}$ Laboratory of Nutrition \& Nutritional Biochemistry, Department of Biochemistry, University of Yaounde I, Cameroon, ${ }^{2}$ Gateway Health Alliances, Inc., 4769 Mangels Blvd, Fairfield, CA 94534 USA, ${ }^{3}$ University of Mississippi, University, MS 38677 USA and ${ }^{4}$ Next Pharmaceuticals, 360 Espinosa Road, Salinas, CA 93907 USA

Email: Julius Oben - juliusoben@hotmail.com; Ebangha Enonchong - juliusoben@hotmail.com; Shil Kothari - shilkothari@hotmail.com; Walter Chambliss - chamblissw@aol.com; Robert Garrison - ddolnick@nextpharmaceuticals.com;

Deanne Dolnick* - ddolnick@nextpharmaceuticals.com

* Corresponding author

Published: 14 August 2009

Nutrition Journal 2009, 8:38 doi:10.1 186/1475-289/-8-38

This article is available from: http://www.nutritionj.com/content/8/I/38

(C) 2009 Oben et al; licensee BioMed Central Ltd.

This is an Open Access article distributed under the terms of the Creative Commons Attribution License (http://creativecommons.org/licenses/by/2.0), which permits unrestricted use, distribution, and reproduction in any medium, provided the original work is properly cited.

\begin{abstract}
Background: The objective of this clinical study was to assess the potential benefit of a dietary supplement, NP 06-I, on joint health in overweight and normal weight adults diagnosed with osteoarthritis.

Methods: An 8-week placebo-controlled, randomized, double-blind study was conducted with four groups comparing the effects of NP 06-I to placebo on overweight and normal weight subjects diagnosed with primary osteoarthritis of the knee. NP 06-I (a combination of two botanical extracts; Phellodendron amurense bark and Citrus sinensis peel) or matching placebo were given in a dose of two capsules (370 mg each) twice daily. The outcome measures were the Lequesne Algofunctional Index (LAI) for joint pain and movement as well as biomarkers of inflammation (C-reactive protein [CRP] and erythrocyte sedimentation rate [ESR]).

Results: Eighty (80) subjects were enrolled and 45 subjects completed the study. No serious adverse events were reported. The mean total LAI scores at baseline for the four groups ranged from II.4 to I2.4 (SD I.2 to 2.4). Treatment for 8 weeks resulted in a statistical improvement in the LAI score in the overweight treatment group compared to placebo $(6.3 \pm 2.3$ vs II $8 \pm 1.5 ; p<0.000 I)$. At 8 weeks, a similar result was observed in the normal weight groups $(7.7 \pm 1.4$ vs $9.9 \pm 0.9 ; p<0.000 \mathrm{I})$. There was a reduction in CRP levels with treatment in the overweight treatment group at 8 weeks $(-0.62 \pm 0.2 ; 49 \%)$ compared to baseline $(p<0.00 \mathrm{I})$ and to placebo $(\mathrm{p}<$ $0.00 \mathrm{I}$ ). For the normal weight participants, there were significant reductions in CRP compared to baseline, but not to the matched placebo group. Both overweight and normal weight treatment groups lost a significant amount of weight compared to their placebo groups. The overweight treatment group lost an average of $5 \%$ body weight after 8 weeks. There was no significant change in ESR in any of the groups.
\end{abstract}

Conclusion: In this pilot study, NP 06-I had beneficial effects on symptoms of osteoarthritis of the knee as measured using LAI scores and had anti-inflammatory effects as measured using CRP. Administration of NP 06-I was also associated with weight loss, which may have been a contributing factor to the other benefits. 


\section{Background}

Osteoarthritis, the most common form of arthritis, is characterized by degradation of articular cartilage, which manifests as joint pain and reduced mobility. The origin of the disease is unknown but obesity, joint injury, metabolic diseases, bone and joint malfunctions, genetic factors and age have been implicated. Therapies for osteoarthritis include weight control, physiotherapy and pharmacological agents. Conventional drug treatments include analgesics, anti-inflammatory agents, disease modifying therapies, hyaluronic acid, intra-articular glucocorticoids and topical analgesic/anti-inflammatory agents [1]. More recently there has been a focus on nutritional support. Recent systematic reviews highlight the scientific evidence for potential nutritional and herbal preparations for those with osteoarthritis [2,3].

The subject of this study, NP 06-1 (Citrofen ${ }^{\circledR}$, Next Pharmaceuticals, Salinas, CA), is a proprietary product consisting of a blend of extracts of Phellodendron amurense tree bark and Citrus sinensis (orange) peel standardized to berberine and polymethoxylated flavones, respectively.

Next Pharmaceuticals has previously conducted bioassay guided research on Phellodendron amurense tree bark resulting in a proprietary extract that has demonstrated analgesic and anti-inflammatory activity both in vitro and in vivo, in unpublished studies. Following this research, a product was developed (Nexrutine ${ }^{\circledast}$ ) that provided relief from pain and/or inflammation associated with overexertion (i.e. sore muscles, sore joints, stiff joints) and osteoarthritis of the knee in unpublished open-label clinical studies.

Citrus sinensis (orange) peel extracts contain bioflavonoids, including polymethoxylated flavones (PMFs). The latter compounds are known to be anti-inflammatory and to have antioxidant and hypolipidemic effects [4-6]. Next Pharmaceuticals has licensed US Patent Nos. 6,184,246 and $6,987,125$ obtained by the USDA for actions described by PMFs.
NP 06-1 was formulated with the goal of combining the beneficial effects of both the phellodendron and orange peel extracts. The primary objective of this clinical study was to study the safety and efficacy of NP 06-1 in the management of joint pain and mobility caused by osteoarthritis of the knee. Secondary objectives were to study the effects on biomarkers related to inflammation. Both overweight and normal weight subjects were studied to determine whether or not there might be a difference in benefits to these two groups. A portion of this clinical study that examined the potential benefits from NP 06-1 on cardiovascular health has recently been published [7]. The published data showed that NP 06-1 had a favorable effect on lipid levels: lowering plasma triglycerides and LDL-cholesterol, as well as increasing HDL-cholesterol levels. NP 06-1 also had a favorable effect on blood pressure and fasting glucose levels (the latter only in the overweight group).

\section{Methods \\ Study Design}

The study was a placebo-controlled, randomized, doubleblind study with four groups. Forty overweight and forty normal weight subjects were enrolled into either treatment or placebo groups, with twenty subjects in each group as shown in Table 1. Subjects were recruited via advertisements at the University of Yaounde I Teaching Hospital, the Djongolo Baptist Hospital and through the public media. The IRB of the Faculty of Medicine and Biomedical Sciences of the University of Yaounde in Cameroon approved the study and all subjects signed an informed consent. Dr. Julius Oben, Associate Professor of Nutritional Biochemistry at the University of Yaounde I, Cameroon was the principal investigator.

To be included in the study, participants needed to be adult men and women, 25 to 60 years of age, diagnosed with primary osteoarthritis of the target knee using the American College of Rheumatology guidelines [8] by the treating physician and confirmed by the clinical investigator, and with a BMI between $25 \mathrm{~kg} / \mathrm{m}^{2}$ to $40 \mathrm{~kg} / \mathrm{m}^{2}$ (over-

Table I: Study Groups.

\begin{tabular}{cllc}
\hline Group \# & BMI Category & Treatment & Enrollment (Completed) \\
\hline OP & Overweight* & Placebo & $20(13)$ \\
\hline OT & Overweight* & Active & $20(14)$ \\
\hline NP & Normal weight $\dagger$ & Placebo & $20(7)$ \\
\hline NT & Normal weight $\dagger$ & Active & $20(11)$ \\
\hline
\end{tabular}

*BMI $25 \mathrm{~kg} / \mathrm{m}^{2}-40 \mathrm{~kg} / \mathrm{m}^{2}$ +BMI $18.9 \mathrm{~kg} / \mathrm{m}^{2}-24.9 \mathrm{~kg} / \mathrm{m}^{2}$

The number of subjects initially enrolled is listed; with the number that completed the study in parenthesis. 
weight groups) or $18.9 \mathrm{~kg} / \mathrm{m}^{2}$ to $24.9 \mathrm{~kg} / \mathrm{m}^{2}$ (normal weight groups).

The exclusion criteria were: morbid obesity BMI $>40 \mathrm{~kg} /$ $\mathrm{m}^{2}$, diagnosis of rheumatoid arthritis, joint replacement in either knee, unable to walk without assistance, enrollment in another clinical study in the past 6 months, pregnancy, active infection, autoimmune disease, AIDS, HIV, active hepatitis, active malignancy, and diabetes requiring daily insulin management.

Subjects were instructed to avoid taking analgesics for a 5 day period prior to enrollment and during the study. Subjects were also instructed to stay with their normal exercise and diet regimens.

\section{Study Products and Administration}

NP 06-1 is a proprietary product of Next Pharmaceuticals, Salinas, California. It is a blend of Phellodendron amurense (Rupr.) [Rutaceae] tree bark extract standardized to a minimum of $50 \%$ berberine and Citrus sinensis (L.) Osbeck [Rutaceae] peel extract standardized to a minimum of $30 \%$ polymethoxylated flavones (PMF). Berberine and PMFs were chosen as representative chemical constituents because they have demonstrated biological activity. Next Pharmaceuticals has licensed US Patent Nos. 6,184,246 and $6,987,125$ obtained by the USDA for biological activity of PMFs.

Subjects were allocated into groups using a random number table and instructed to take two NP 06-1 capsules (370 mg formula per capsule) or matching placebo (identical red two-piece hard shell capsules) with food in the morning and at night ( 4 capsules per day) for a total of 8 weeks.

\section{Study Variables}

The study variables were: physical pain and joint movement as measured by the total score on the standardized LAI questionnaire [9], as well as biomarkers for inflammation: CRP and ESR. The LAI scale (algofunctional indices) is a validated instrument for evaluating therapies for osteoarthritis. The LAI score is the sum of the responses to 10 questions regarding pain/discomfort, maximum distance walked and activities of daily living. The subjects were asked to respond to each question using a 5 point Likert scale $(0,0.5,1.0,1.5$ or 2.0 ; where 0 is without and 2 is with difficulty/discomfort). The LAI questionnaire was completed by each subject at baseline, at 4 weeks and at 8 weeks. Blood samples $(8 \mathrm{ml})$ were collected by venous puncture at times 0,4 and 8 weeks. Subjects were requested to come to the study center on the mornings of each site visit after a 12-hour fast. CRP was measured using the Dade Turbitimer. ESR was measured using the Westergren method. Body weight was determined in 12 - hour fasted subjects using a Tanita ${ }^{\mathrm{TM}}$ scale and height was measured using a stadiometer.

\section{Safety Assessment}

Subjects were given three emergency telephone numbers to contact during the conduct of the study, if they had any adverse events or other concerns related to the study. Each subject was interviewed during site visits to solicit information on possible adverse effects they might have encountered. Participants were instructed to inform the investigator, if the reason for dropping out of the study was due to adverse effects.

\section{Statistical Analysis}

Data were analyzed using SAS version 9.2 (SAS Institute, 1999). Descriptive statistics were run for each group (Placebo and NP 06-1) and each time point (baseline, 4 weeks and 8 weeks). Initially, an analysis of variance model was used to determine whether or not the groups and times differed in baseline scores. No differences were found. An analysis of variance model, with repeated measures, was used to compare changes of physiological measures over the trial period and between groups. First, we examined models with all 2-way and 3-way interactions. Since the 3way interactions were found significant, we examined each weight group separately. For each weight group, we used an analysis of variance model with treatment (Placebo vs and NP 06-1) as a fixed effect and time (4 weeks vs 8 weeks) as a repeated measure to determine the effects of NP 06-1.

\section{Results}

Eighty (80) subjects were enrolled in the study and randomized into four groups designated OP (Overweight/Placebo), OT (Overweight/Treatment), NP (Normal weight/ Placebo) and NT (Normal weight/Treatment) (Table 1). Forty five (45) subjects completed the study and the reasons given by the dropouts are given in Table 2 . The dropouts were fairly evenly distributed among the groups, with the exception of Group NP which lost nearly twice as many participants as the other groups. The majority of Group NP dropouts cited no improvement in their condition as their reason for dropping out. There is no indication that their demographics were different from those who stayed in the study. No serious adverse events were reported by any of the dropouts or by those who completed the study. The following results were compiled using data from those who completed the study.

\section{LAI Scores}

The mean total LAI scores at baseline for the four groups ranged from 11.4 to 12.4 (standard deviations 1.2 to 2.4 ) with no statistical differences between them $(\mathrm{p}=0.43)$ (Table 3). Comparison of the overweight groups (OT vs. OP) showed a significant decrease in LAI scores in the 
Table 2: Reasons given by Subjects for dropping out of the Study.

\begin{tabular}{|c|c|c|}
\hline Group \# & Number of Subjects & Reason for drop out \\
\hline \multirow[t]{4}{*}{ OP } & 2 & Reported no improvement in their condition \\
\hline & 1 & Nausea and vomiting; Malaria attack \\
\hline & 1 & Moved out of town \\
\hline & 3 & No reason given \\
\hline \multirow[t]{4}{*}{ OT } & 1 & Reported improvement as too slow \\
\hline & 1 & Tested positive for hepatitis \\
\hline & 1 & Moved out of town \\
\hline & 3 & No reason given \\
\hline \multirow[t]{5}{*}{ NP } & 7 & Reported no improvement in their condition \\
\hline & 1 & Nausea \\
\hline & 1 & Started weight management program \\
\hline & 1 & Started fasting and stopped treatment \\
\hline & 3 & No reason given \\
\hline \multirow[t]{4}{*}{ NT } & 1 & Malaria attack \\
\hline & 1 & Nausea \\
\hline & 1 & Started fasting and stopped treatment \\
\hline & 6 & No reason given \\
\hline
\end{tabular}

treatment group compared to the placebo at 4 weeks $(8.2$ \pm 2.7 vs $11.7 \pm 1.7)$ and at 8 weeks $(6.3 \pm 2.3$ vs $11.8 \pm 1.5)$ (both p <0.0001) (Table 4). For Group OT, there were statistically significant decreases in LAI scores at 4 weeks ($3.5 \pm 2.2)$ and 8 weeks $(-5.4 \pm 2.2)$ compared to baseline (both $\mathrm{p}<0.0001)$ (Table 3 ). There was also a difference between the 4 and 8 week time periods ( $p=0.002)$. In contrast there were no statistically significant changes from baseline for Group OP.

Comparison of normal weight groups (NT vs. NP) also showed a significant decrease in LAI scores in the treatment compared to the placebo group at 4 weeks $(7.6 \pm 1.9$ vs $10.3 \pm 1.5)$ and 8 weeks $(7.7 \pm 1.4$ vs $9.9 \pm 0.9$ (both $p$ $<0.0001)$. For Group NT there were statistically significant decreases in LAI scores compared to baseline at 4 weeks $(-3.7 \pm 2.1)$ and 8 weeks $(-3.7 \pm 1.7)$ (both $\mathrm{p}<$ $0.0001)$. There was also a difference between the 4 and 8 week time periods $(\mathrm{p}=0.012)$. For Group NP there were significant differences between baseline and 4 weeks $(-1.4$ $\pm 2.8 ; \mathrm{p}=0.019)$ and between baseline and 8 weeks $(-1.9$ $\pm 2.1 ; \mathrm{p}<0.0001)$. No significant difference was found between the 4 and 8 week time periods.

Multivariate analysis showed a treatment effect for NP 061 in both the overweight and normal weight groups $(\mathrm{p}<$ 
Table 3: LAI data.

\begin{tabular}{cccccc}
\hline Group & Baseline & 4 weeks & 8 weeks & $\Delta 0-4$ wks & $\Delta 0-8$ wks \\
\hline OP & $12.4 \pm 1.3$ & $11.7 \pm 1.7$ & $11.8 \pm 1.5$ & $-0.7 \pm 1.6$ & $-0.6 \pm 1.7$ \\
\hline OT & $11.7 \pm 1.5$ & $8.2 \pm 2.7$ & $6.3 \pm 2.3$ & $-3.5 \pm 2.2^{*}$ & $-5.4 \pm 2.2^{*}$ \\
\hline NP & $11.7 \pm 2.4$ & & & $-1.4 \pm 2.8^{*}$ & $-1.9 \pm 2.1^{*}$ \\
\hline NT & $11.4 \pm 1.2$ & $10.3 \pm 1.5$ & $9.9 \pm 0.9$ & $-3.7 \pm 2.1^{*}$ & $-3.7 \pm 1.7^{*}$ \\
\hline
\end{tabular}

Values at baseline, 4 weeks and 8 weeks are listed as means \pm standard deviation. Mean changes $(\Delta) \pm$ standard deviation after 4 weeks and 8 weeks compared to baseline are listed in the last two columns. A negative value indicates a decrease in value. Values* are significant comparisons ( $<0.05)$ based on analysis of variance model results comparing changes from baseline to 4 weeks or 8 weeks. Groups are as follows: OP $=$ overweight/ placebo, OT = overweight/treatment, NP = normal weight/placebo and NT = normal weight/treatment.

0.0001 and 0.003 , respectively), with no difference in the effect between the overweight and normal weight groups (weight by treatment interaction).

\section{Biomarkers of Inflammation}

The mean total CRP measurements at baseline for the four groups ranged from 0.8 to $1.3 \mathrm{mg} / \mathrm{L}$ (Table 5). There were no significant differences between the groups at baseline. Comparison of overweight groups showed a significant difference in CRP between OT and OP groups at 4 and 8 weeks ( $p<0.05$ and $\mathrm{p}<0.001$, respectively). For Group OT, there were significant decreases in CRP at 4 weeks ($0.35 \pm 0.2 ; 28 \%)$ and 8 weeks $(-0.62 \pm 0.2 ; 49 \%)$ compared to baseline (both $\mathrm{p}<0.001$ ). There were smaller, but significant, changes from baseline for Group OP. Comparison of normal weight groups showed no significant differences in CRP between treatment and placebo. For Group NT there were significant decreases in CRP over

Table 4: Inter-Group Analysis Comparing the Paired Treatment to PlaceboGroups.

\begin{tabular}{ccccc}
\hline & \multicolumn{2}{c}{ Group OP vs. Group 0T } & \multicolumn{2}{c}{ Group NP vs. Group NT } \\
\hline & 4 weeks & $\mathbf{8}$ weeks & 4 weeks & $\mathbf{8}$ weeks \\
\hline LAI & $<0.0001$ & $<0.0001$ & $<0.0001$ & $<0.0001$ \\
\hline CRP & $<0.05$ & $<0.001$ & NS & NS \\
\hline ESR & NS & NS & $<0.05$ & NS \\
\hline Weight & $<0.01$ & $<0.001$ & $<0.05$ & $<0.01$ \\
\hline BMI & NS & NS & NS & NS
\end{tabular}

Based on analysis of variance F-statistics, $P$-values are for comparisons between overweight placebo (OP) and overweight treatment (OT) groups and between normal weight placebo (NP) and normal weight treatment (NT) groups at 4 and 8 weeks. NS $=$ not significant. time; at 4 weeks $(-0.31 \pm 0.2 ; 27 \%)$ and 8 weeks $(-0.51 \pm$ $0.2 ; 44 \%$ ) compared to baseline (both $\mathrm{p}<0.001$ ). In contrast there were no significant changes from baseline for Group NP. There were no significant changes in ESR in any of the groups (Table 5).

\section{Weight/BMI}

In Group OT, the subjects lost a significant amount of weight and their BMI was reduced compared to the start of the study ( $\mathrm{p}<0.001$ at 4 and 8 weeks) (Table 6). They lost an average of $2.5 \mathrm{~kg}$ (5.5 pounds, $3.1 \%$ of body weight) after 4 weeks and an average of $4.2 \mathrm{~kg}(9.2$ pounds, $5.1 \%$ of body weight) after 8 weeks. There were smaller, but significant, changes in weight and BMI from baseline for Group OP. Comparisons of weight loss between overweight groups revealed that the treatment group lost on average 3.7 times as much weight as the placebo group at 8 weeks ( 9.2 pounds versus 2.5 pounds) (p $<0.001)$. However, in comparison, the changes in BMI between the two overweight groups were not significant.

In Group NT there was a significant decrease in weight and BMI at 8 weeks $(\mathrm{p}<0.05)$ compared to baseline. At 8 weeks, this group lost an average of $1.18 \mathrm{~kg}$ (2.6 pounds, $1.8 \%$ of body weight). Group NP also had a significant loss of weight and BMI after 8 weeks compared to the start of the study $(\mathrm{p}<0.01)$. Comparisons of weight loss between normal weight groups revealed that the treatment group lost on average 1.5 times as much weight as the placebo group at 8 weeks (2.6 pounds versus 1.7 pounds) ( $\mathrm{p}<0.01)$. There was no significant difference in change in BMI between the normal weight treatment and placebo groups.

\section{Discussion}

LAI scores (algofunctional indices) have been validated for use in assessing the severity of osteoarthritis of the hip and knee [9]. LAI scores, which incorporate points for 
Table 5: Markers of Inflammation: CRP \& ESR.

\begin{tabular}{|c|c|c|c|c|c|}
\hline $\begin{array}{c}\text { Group } \\
\text { Variable }\end{array}$ & Baseline & 4 weeks & 8 weeks & $\Delta 0-4$ wks & $\Delta 0-8$ wks \\
\hline \multicolumn{6}{|l|}{ OP } \\
\hline CRP (mg/L) & $1.19 \pm 0.26$ & $1.02 \pm 0.27$ & $1.08 \pm 0.25$ & $-0.17 \pm 0.2^{*}$ & $-0.11 \pm 0.07^{*}$ \\
\hline $\operatorname{ESR}(\mathrm{mm} / \mathrm{h})$ & $13.6 \pm 2.5$ & $13.5 \pm 2.0$ & $13.6 \pm 1.7$ & $-0.10 \pm 2.4$ & $0.0 \pm 2.4$ \\
\hline \multicolumn{6}{|l|}{ OT } \\
\hline CRP (mg/L) & $1.33 \pm 0.20$ & $0.95 \pm 0.11$ & $0.68 \pm 0.14$ & $-0.35 \pm 0.2^{*}$ & $-0.62 \pm 0.2 *$ \\
\hline $\mathrm{ESR}(\mathrm{mm} / \mathrm{h})$ & $12.7 \pm 0.9$ & $13.0 \pm 2.1$ & $12.9 \pm 1.6$ & $0.31 \pm 2.4$ & $0.38 \pm 1.8$ \\
\hline \multicolumn{6}{|l|}{$\mathbf{N P}$} \\
\hline CRP (mg/L) & $0.76 \pm 0.19$ & $0.76 \pm 0.19$ & $0.68 \pm 0.18$ & $0.04 \pm 0.1$ & $-0.08 \pm 0.1$ \\
\hline ESR $(\mathrm{mm} / \mathrm{h})$ & $12.5 \pm 1.4$ & $12.8 \pm 1.7$ & $12.8 \pm 1.3$ & $0.30 \pm 1.0$ & $0.30 \pm 1.1$ \\
\hline \multicolumn{6}{|l|}{ NT } \\
\hline CRP (mg/L) & $1.15 \pm 0.22$ & $0.84 \pm 0.13$ & $0.64 \pm 0.50$ & $-0.31 \pm 0.2^{*}$ & $-0.51 \pm 0.2 *$ \\
\hline ESR $(\mathrm{mm} / \mathrm{h})$ & $13.1 \pm 1.2$ & $13.5 \pm 1.7$ & $13.3 \pm 0.9$ & $0.36 \pm 2.5$ & $0.18 \pm 1.3$ \\
\hline
\end{tabular}

Values at baseline, 4 weeks and 8 weeks are listed as means \pm standard deviation. Mean changes $(\Delta) \pm$ standard deviation after 4 weeks and 8 weeks compared to baseline are listed in the last two columns. A negative value indicates a decrease in value and a positive number represents an increase in value. Values* are significant comparisons $(<0.05)$ based on analysis of variance model results comparing changes from baseline to 4 weeks or 8 weeks. Groups are as follows: OP = overweight/placebo, OT = overweight/treatment, NP = normal weight/placebo and NT = normal weight/ treatment.

pain, maximum distance walked and activities of daily living, typically range from 1 to 14 . Scores of 1 to 4 indicate a minor handicap while a score of 14 indicates an extremely severe handicap. Clinical studies used to assess the potential benefits of drugs on osteoarthritis typically start with subjects who have scores of 9 to 11 (SD 2.3 to 3.8). Upon administration of non-steroidal anti-inflammatory drugs (NSAIDS) for one or two weeks, those scores usually decrease by 2.5 to 4 points: decreases of approximately 25 to $40 \%$. In this study, the groups had mean scores within the range of 11 to 13 (SD 1.2 to 2.4) at baseline, which indicates a very severe handicap from osteoarthritis of the knee. After 8 weeks there was a decrease of $3.7 \pm 2.1$ points in the normal weight treatment group and a decrease of $5.4 \pm 2.2$ points in the overweight treatment group. The degree of improvement observed with NP 061 is comparable to that reported for NSAIDS.

CRP is an acute phase protein that is produced by the liver during an inflammatory reaction. Serum CRP levels are often higher in those with osteoarthritis and are associated with joint pain, as well as the severity and progression of the disease $[10,11]$. Recent studies have indicated that the correlation between CRP levels and osteoarthritis involves other variables besides the disease state [12]. Body weight has been determined to influence CRP levels due to mechanical effects, as well as the production of inflammatory mediators by adipocytes. A systematic review of the literature covering weight reduction in obese subjects diagnosed with osteoarthritis of the knee concluded that the disability associated with the disease could be significantly improved with a loss of over $5.1 \%$ body weight [13]. In contrast with the improvement in disability, loss of body weight did not necessarily predict relief from pain. Another systematic review reported that weight loss in the general adult population is associated with reductions in CRP levels. This analysis concluded that for each $1 \mathrm{~kg}$ of weight loss, the mean change in CRP level was decreased by $0.13 \mathrm{mg} / \mathrm{L}$ (weighted Pearson correlation, $\mathrm{r}=$ $0.85)$ [14]. A study investigating the correlation between CRP and osteoarthritic disease progression found that subjects with inflammatory infiltrates within the synovial membrane had a mean CRP level of $4.7 \pm 5.0 \mathrm{mg} / \mathrm{L}$, while those without infiltrates had a mean CRP level of $1.7 \pm 3.6$ [11]. 
Table 6: Body Weight, BMI.

\begin{tabular}{|c|c|c|c|c|c|}
\hline Group & Baseline & 4 weeks & 8 weeks & $\Delta 0-4$ wks & $\Delta 0-8$ wks \\
\hline \multicolumn{6}{|l|}{ OP } \\
\hline Weight (kg) & $85.0 \pm 12.7$ & $83.9 \pm 12.6$ & $83.8 \pm 12.2$ & $-1.1 \pm 0.96^{*}$ & $-1.2 \pm 1.3^{*}$ \\
\hline BMI $\left(\mathrm{kg} / \mathrm{m}^{2}\right)$ & $31.1 \pm 4.2$ & $30.7 \pm 4.2$ & $30.7 \pm 4.1$ & $-0.4 \pm 0.59 *$ & $-0.4 \pm 0.32^{*}$ \\
\hline \multicolumn{6}{|l|}{ OT } \\
\hline Weight (kg) & $81.7 \pm 9.3$ & $79.2 \pm 9.1$ & $77.5 \pm 8.3$ & $-2.5 \pm 1.09 *$ & $-4.2 \pm 1.24^{*}$ \\
\hline BMI $\left(\mathrm{kg} / \mathrm{m}^{2}\right)$ & $31.1 \pm 3.7$ & $30.2 \pm 3.7$ & $29.5 \pm 3.4$ & $-0.9 \pm 0.11^{*}$ & $-1.6 \pm 0.11^{*}$ \\
\hline \multicolumn{6}{|l|}{$\mathbf{N P}$} \\
\hline Weight (kg) & $67.1 \pm 3.6$ & $66.8 \pm 3.8$ & $66.5 \pm 3.8$ & $-0.3 \pm 0.60$ & $-0.6 \pm 0.34^{*}$ \\
\hline BMI $\left(\mathrm{kg} / \mathrm{m}^{2}\right)$ & $24.0 \pm 1.6$ & $23.9 \pm 1.6$ & $23.8 \pm 1.6$ & $-0.1 \pm 0.05$ & $-0.2 \pm 0.04 *$ \\
\hline \multicolumn{6}{|l|}{ NT } \\
\hline Weight (kg) & $67.0 \pm 9.7$ & $66.5 \pm 8.9$ & $65.8 \pm 8.7$ & $-0.5 \pm 1.08$ & $-1.2 \pm 1.3^{*}$ \\
\hline BMI $\left(\mathrm{kg} / \mathrm{m}^{2}\right)$ & $24.8 \pm 2.6$ & $24.7 \pm 2.3$ & $24.4 \pm 2.3$ & $-0.1 \pm 0.05$ & $-0.4 \pm 0.08^{*}$ \\
\hline
\end{tabular}

Values at baseline, 4 weeks and 8 weeks are listed as means \pm standard deviation. Mean changes $(\Delta) \pm$ standard deviation after 4 weeks and 8 weeks compared to baseline are listed in the last two columns. A negative value indicates a decrease in value and a positive number represents an increase in value. Values* are significant comparisons $(<0.05)$ based on analysis of variance model results comparing changes from baseline to 4 weeks or 8 weeks. Groups are as follows: OP = overweight/placebo, OT = overweight/treatment, NP = normal weight/placebo and NT = normal weight/ treatment.

In the present study, the mean levels of CRP at baseline for the four groups ranging from 0.76 to $1.3 \mathrm{mg} / \mathrm{L}$ indicated a relatively low level of inflammation. In spite of this, there was a reduction in CRP for the overweight participants with treatment compared to placebo. There was no comparative treatment effect for the normal weight participants. However there was an effect of treatment over time for both the overweight and normal weight groups. The overweight treatment group experienced a weight loss of $5.1 \%$ after 8 weeks. The normal weight subjects also lost body weight, but to a lesser degree. It appears from current literature, that the decreases in CRP in this study may have been influenced by loss in body weight $[13,14]$. Also the reduction in weight may have had an influence in the reduction in LAI scores [13].

NP 06-1 contains standardized amounts of berberine, a constituent of the Phellodendron extract, and PMFs from citrus. Both of these components have demonstrated antiinflammatory activity. Plant extracts containing berberine have traditionally been used in rheumatic and other chronic inflammatory diseases [15]. In support of the traditional use, extracts containing berberine have demonstrated anti-inflammatory activity in animal models
$[16,17]$. In vitro studies indicate that berberine inhibits the production of inflammatory mediators prostaglandin $\mathrm{E}_{2}$ and interleukin (IL)-12 [18,19]. Berberine also reduced the levels of expression of mRNA for pro-inflammatory cytokines including tumor necrosis factor (TNF)-alpha, IL-6, CRP and haptoglobin [20].

Berberine has also been shown to reduce body weight in mice and rats [21]. Histological studies indicated that berberine reduced adipocyte size but not cell number. Measurements of metabolic gene expression in adipose and muscle tissue indicated that berberine up regulated genes involved in energy expenditure and down regulated the expression of genes involved in lipogenesis. In vitro studies determined that berberine increased AMP-activated protein kinase (AMPK) activity in 3T3-L1 adipocytes and L6 myotubes, as well as reduced lipid accumulation in 3T3-L1 cells. In addition, berberine reduced secretion of leptin, which regulates appetite and energy metabolism, from 3T3-L1 adipocytes.

Citrus PMFs have been reported to exhibit anti-inflammatory activity in vivo and in vitro. PMFs regulated levels of adipocytokines through suppression of TNF-alpha, inter- 
feron-gamma, IL-1 beta and IL-6 expression [22]. PMFs also suppressed TNF-alpha expression by monocytes, perhaps due to inhibition of phosphodiesterase activity [23].

This pilot study has a number of weaknesses. The most obvious weakness is the number of dropouts. There was no clear evidence of difference or bias in the demographics of the dropouts compared to those who stayed in the study; however, there was insufficient information to run an analysis of the two groups. Care must be taken with respect to dropouts when a full blown study is conducted. We also acknowledge that we have no information on patient compliance with treatment protocol.

In summary, it appears from the results of this pilot study that NP 06-1 may benefit subjects with osteoarthritis of the knee through an anti-inflammatory action as well as through a loss of body weight. In addition to increased osteoarthritis-related disability, obesity is correlated with an array of conditions associated with a high risk of diabetes, hypertension and/or cardiovascular disease called metabolic syndrome [24]. Symptoms of metabolic syndrome include abdominal obesity, dyslipidemia and elevated blood pressure and insulin resistance.

We have previously reported that administration of NP 06-1 is associated with a general improvement in lipid levels, with reductions in triglycerides and low-density lipoprotein (LDL)-cholesterol and an increase in high-density lipoprotein (HDL)-cholesterol [7]. In that same report, we also reported treatment-related decreases in blood pressure and a decrease in fasting glucose levels in an overweight population. Thus additional studies are indicated to explore the use of NP 06-1 for osteoarthritis and for metabolic syndrome.

\section{Conclusion}

NP 06-1 has been shown in a placebo-controlled, randomized, double-blind pilot clinical study to offer several potential health benefits in normal and overweight subjects with osteoarthritis of the knee. These potential benefits include improvement of knee joint pain/flexibility as measured by the LAI scores and a reduction in inflammation as measured using CRP levels. Treatment-induced weight loss may have been a contributing factor to these improvements. NP 06-1 was administered to the trial participants in a dose of 4 capsules $(1,480 \mathrm{mg})$ per day for 8 weeks without significant adverse events.

\section{Competing interests}

This study was sponsored by Next Pharmaceuticals. Mr. Garrison was President and Miss Dolnick are employed by Next Pharmaceuticals. Dr. Chambliss and Mr. Kothari were compensated as consultants to Next Pharmaceuticals

\section{Authors' contributions}

All authors were involved in the design of the study, as well as analysis and interpretation of the data. The study was carried out by Dr. Oben, the Principal Investigator and Miss Enonchong, his assistant. All authors read and approved the final manuscript.

\section{Acknowledgements}

We thank Marilyn Barrett $\mathrm{PhD}$, who provided medical writing on behalf of Next Pharmaceuticals. We also acknowledge Ginny Gildengorin PhD, who conducted the statistical analyses on the LAI data.

\section{References}

I. Bijlsma JW, Knahr K: Strategies for the prevention and management of osteoarthritis of the hip and knee. Best Pract Res Clin Rheumatol 2007, 2 I(I):59-76.

2. Ameye LG, Chee WS: Osteoarthritis and nutrition. From nutraceuticals to functional foods: a systematic review of the scientific evidence. Arthritis Res Ther 2006, 8(4):RI27.

3. Long L, Soeken K, Ernst E: Herbal medicines for the treatment of osteoarthritis: a systematic review. Rheumatology 200I, 40:779-793.

4. Kurowska EM, Manthey JA: Hypolipidemic effects and absorption of citrus polymethoxylated flavones in hamsters with diet-induced hypercholesterolemia. J Agric Food Chem 2004, 52( ( 0):2879-86.

5. Kurowska EM, Manthey JA, Casaschi A, Theriault AG: Modulation of Hepg 2 cell net apolipoprotein B secretion by the Citrus polymethoxyflavone, tangeretin. Lipids 2004, 39(2):I43-I5I.

6. Whitman SC, Kurowska EM, Manthey JA, Daugherty A: Nobiletin, a citrus flavonoid isolated from tangerines, selectively inhibits class A scavenger receptor-mediated metabolism of acetylated LDL by mouse macrophages. Atherosclerosis 2005, I 78(I):25-32.

7. Oben J, Enonchong E, Kothari S, Chambliss W, Garrison R, Dolnick $\mathrm{D}$ : Phellodendron and Citrus extracts benefit cardiovascular health in osteoarthritis patients: a double-blind, placebocontrolled pilot study. Nutr J 2008, 20(7): 16.

8. Altman R, Asch E, Bloch D, Bole G, Borenstein D, Brandt K, et al.: The American College of Rheumatology criteria for the classification and reporting of osteoarthritis of the knee. Arthritis Rheum 1986, 29:1039-1049.

9. Lequesne MG: The algofunctional indices for hip and knee osteoarthritis. J Rheumatol 1997, 24:779-8I.

10. Wolfe F: The C-reactive protein but not erythrocyte sedimentation rate is associated with clinical severity in patients with osteoarthritis of the knee or hip. J Rheumatol 1997, 24(8): |486-8.

II. Pearle AD, Scanzello CR, George S, Mandl LA, Dicarlo EF, Peterson M, Sculco TP, Crow MK: Elevated high-sensitivity C-reactive protein levels are associated with local inflammatory findings in patients with osteoarthritis. Osteoarthr Cartil 2007, I5(5):5 1 6-23.

12. Kraus VB, Stabler TV, Luta G, Renner JB, Dragomir AD, Jordan JM: Interpretation of serum C-reactive protein (CRP) levels for cardiovascular disease risk is complicated by race, pulmonary disease, body mass index, gender, and osteoarthritis. Osteoarthritis Cartilage 2007, I 5(8):966-7I.

13. Christensen R, Bartels EM, Astrup A, Bliddal H: Effect of weight reduction in obese patients diagnosed with knee osteoarthritis: a systematic review and meta-analysis. Ann Rheum Dis 2007, 66(4):433-9.

14. Selvin E, Paynter NP, Erlinger TP: The effect of weight loss on Creactive protein: a systematic review. Arch Intern Med 2007, I67(1):31-9.

15. Ivanovska N, Philipov S: Study on the anti-inflammatory action of Berberis vulgaris root extract, alkaloid fractions and pure alkaloids. Int J Immunopharmacol I996, I 8( I 0):553-6I.

16. Ivanovska N, Philipov S, Hristova M: Influence of berberine on Tcell mediated immunity. Immunopharmacol Immunotoxicol 1999, 2 I (4):77|-86. 
17. Kubo M, Matsuda H, Tokuoka K, Ma S, Shiomoto H: Anti-inflammatory activities of methanolic extract and alkaloidal components from Corydalis tuber. Biol Pharm Bull 1994, I 7(2):262-5.

18. Kuo CL, Chi CW, Liu TY: The anti-inflammatory potential of berberine in vitro and in vivo. Cancer Lett 2004, 203(2): 127-37.

19. Lee DU, Kang YJ, Park MK, Lee YS, Seo HG, Kim TS, Kim CH, Chang KC: Effects of I3-alkyl-substituted berberine alkaloids on the expression of COX-II, TNF-alpha, iNOS, and IL-I 2 production in LPS-stimulated macrophages. Life Sci 2003, 73(II): $1401-12$.

20. Choi BH, Ahn IS, Kim YH, Park JW, Lee SY, Hyun CK, Do MS: Berberine reduces the expression of adipogenic enzymes and inflammatory molecules of 3T3-LI adipocyte. Exp Mol Med. 2006, 38(6):599-605.

2I. Lee YS, Kim WS, Kim KH, Yoon MJ, Cho HJ, Shen Y, Ye JM, Lee CH, Oh WK, Kim CT, Hohnen-Behrens C, Gosby A, Kraegen EW, James $\mathrm{DE}, \mathrm{Kim} \mathrm{JB}$ : Berberine, a natural plant product, activates AMP-activated protein kinase with beneficial metabolic effects in diabetic and insulin-resistant states. Diabetes 2006, 55(8):2256-64.

22. Li RW, Theriault AG, Au K, Douglas TD, Casaschi A, Kurowska EM Mukherjee R: Citrus polymethoxylated flavones improve lipid and glucose homeostasis and modulate adipocytokines in fructose-induced insulin resistant hamsters. Life Sci 2006, 79(4):365-73.

23. Manthey JA, Grohmann K, Montanari A, Ash K, Manthey CL: Polymethoxylated flavones derived from citrus suppress tumor necrosis factor-alpha expression by human monocytes. J Nat Prod 1999, 62(3):44I-4.

24. Cornier MA, Dabelea D, Hernandez TL, Lindstrom RC, Steig AJ, Stob NR, et al.: The metabolic syndrome. Endocr Rev 2008, 29:777-822.

Publish with Biomed Central and every scientist can read your work free of charge

"BioMed Central will be the most significant development for disseminating the results of biomedical research in our lifetime. "

Sir Paul Nurse, Cancer Research UK

Your research papers will be:

- available free of charge to the entire biomedical community

- peer reviewed and published immediately upon acceptance

- cited in PubMed and archived on PubMed Central

- yours - you keep the copyright

Submit your manuscript here:

http://www.biomedcentral.com/info/publishing_adv.asp
BioMedcentral 